\title{
Linking Amyloid and Tau Pathology in Alzheimer's Disease: The Role of Membrane Cholesterol in A $\beta$-Mediated Tau Toxicity
}

\author{
Selina Wray ${ }^{1}$ and Wendy Noble ${ }^{2}$ \\ ${ }^{1}$ Department of Molecular Neuroscience, UCL Institute of Neurology, London WC1N 3BG, United Kingdom, and ${ }^{2}$ Department of Neuroscience, MRC Centre \\ for Neurodegeneration Research, Institute of Psychiatry, King's College London, London SE5 8AF, United Kingdom \\ Review of Nicholson and Ferreira
}

Alzheimer's disease (AD) is characterized by extracellular deposition of $\beta$-amyloid $(\mathrm{A} \beta)$ as senile plaques, as well as intracellular accumulation of hyperphosphorylated, aggregated tau as neurofibrillary tangles. Increased production and/or deposition of $\mathrm{A} \beta$ is thought to precede tangle formation, but evidence suggests that tau pathology is more closely related to neuronal death (reviewed by Hanger et al., 2009). The importance of tau in mediating $\mathrm{A} \beta$ toxicity has been demonstrated by the resistance of tau knock-out neurons to $A \beta$-induced neurotoxicity and the rescue of cognitive impairment in mutant amyloid precursor protein (APP) transgenic mice when crossed with a tau knock-out line (Roberson et al., 2007). However, the mechanistic link between $\mathrm{A} \beta$, disease-associated changes in tau proteins, and cell death remains the subject of intensive research.

Several studies demonstrate that fragments generated by the proteolytic cleavage of tau by caspases and/or calpains play a role in neurodegeneration. Immunohistochemical studies have demonstrated that tau

Received May 6, 2009; revised June 19, 2009; accepted June 27, 2009.

We thank Dr. Diane Hanger and Dr. Patrick Lewis for helpful discussion and editorial comments on this manuscript.

Correspondence should be addressed to Selina Wray, Department of Molecular Neuroscience, UCL Institute of Neurology, Queen Square, London WC1N 3BG,UK.E-mail:S.Wray@ion.ucl.ac.uk.

DOI:10.1523/JNEUROSCI.2234-09.2009

Copyright $\odot$ 2009 Society for Neuroscience $\quad$ 0270-6474/09/299665-03\$15.00/0 cleaved by caspase- 3 is present in neuronal lesions of several tauopathies. Caspase-3cleaved tau fragments show a higher propensity to aggregate than full-length tau, and generation of these fragments precedes alterations in tau conformation, phosphorylation, and aggregation, suggesting cleavage is a relatively early event in neurofibrillary tangle formation (Cotman et al., 2005). Mutant forms of tau that are resistant to caspase-3 cleavage prevent caspase-induced cell death, indicating a direct relationship between the generation of tau fragments and neuronal demise (Cotman et al., 2005). The calciumactivated cysteine protease, calpain, also digests tau, producing several fragments, including a $17 \mathrm{kDa}$-terminal fragment comprising residues $44-228$ of the longest CNS tau isoform that induces apoptosis when exogenously expressed in cell lines. Furthermore, $\mathrm{A} \beta$ activates calpain and increases tau proteolysis in primary neurons (Park and Ferreira., 2005). Thus, proteolytic cleavage of tau may be a key step in $\mathrm{A} \beta$-induced cell death pathways.

In a recent issue of The Journal of $\mathrm{Neu}$ roscience, Nicholson and Ferreira (2009) extend their previous findings (Park and Ferreira, 2005) by showing that $\mathrm{A} \beta$ mediated production of $17 \mathrm{kDa}$ calpaincleaved tau fragments changes increases with neuronal development. The authors cultured hippocampal neurons for 7-12 or 17-21 d in vitro, classing these as immature or mature cultures, respectively, based on differential expression of fetal and adult tau isoforms. Treatment with 10-20 $\mu \mathrm{M}$ preaggregated $\mathrm{A} \beta$ demonstrated that immature neurons were more resistant than mature neurons to $\mathrm{A} \beta$ induced toxicity and that the apparent vulnerability of mature neurons was associated with calpain activation and production of $17 \mathrm{kDa}$ tau species. The authors conclude that, since there is no difference in the susceptibility of fetal and human tau isoforms to cleavage by calpain in vitro, the differences between immature and mature neurons are attributable to the impact of heightened $A \beta$-induced calpain activation in mature neurons.

Membrane cholesterol content regulates the rate of calcium influx and calpain activation in neurons by increasing the activity of glutamatergic receptors and membraneassociated calcium transporters. Nicholson and Ferreira (2009) investigated whether the increased cholesterol content observed in mature neurons induces calpain overactivation in response to $A \beta$. Lowering membrane cholesterol in mature neurons with MBCD (methyl- $\beta$-cyclodextrin) resulted in a transient reversal of the $\mathrm{A} \beta$ induced increases in intraneuronal calcium concentrations and the accompanying calpain activation, generation of $17 \mathrm{kDa}$ tau fragments, and neuronal death. Conversely, increasing membrane cholesterol in imma- 
ture neurons increased their sensitivity to $\mathrm{A} \beta$. The authors conclude that maturationdependent elevations in membrane cholesterol increase the susceptibility of neurons to $\mathrm{A} \beta$-induced calpain activation and tau toxicity.

Nicholson and Ferreira (2009) are the first to suggest an association between membrane cholesterol levels and tau toxicity in the context of $\mathrm{AD}$; however, some of their results raise additional questions, and some conclusions seem premature.

The first, and perhaps most important, issue that needs to be addressed, is the relevance of the $17 \mathrm{kDa}$ tau fragment to $\mathrm{AD}$. Although this fragment has been observed in primary neurons, it has yet to be found in human brain. Calpain-cleaved fragments of tau have been identified in human brain tissue (Mercken et al., 1995), but these are different ( 30 and $36 \mathrm{kDa}$ ) species to those described by Nicholson and Ferreira. In $\mathrm{AD}$, tau forms insoluble paired helical filaments (PHF), and although calpain activity is increased in $\mathrm{AD}$, it has been shown that PHF-tau is less susceptible to calpain degradation than tau from control brain (Mercken et al., 1995). It is possible that the $17 \mathrm{kDa}$ tau species might be generated before PHF formation, but until this species is identified in human brain, it cannot be asserted that its production is an early event in $\mathrm{AD}$.

Second, it is not clear whether calpain cleavage of tau precedes or follows tau phosphorylation. Hyperphosphorylation of tau is a prominent feature in $\mathrm{AD}$ and recent evidence indicates a neurotoxic role of soluble species of phosphorylated tau (reviewed by Hanger et al., 2009). A $\beta$ treatment activates several tau kinases, including cdk5 and GSK-3, both of which can be activated by calpain, and that phosphorylate many residues on PHF-tau (Hanger et al., 2009). Thus, although this is not reported, it is likely that some degree of tau phosphorylation occurs in the model used by Nicholson and Ferreira (2009). The generation of other tau fragments, such as caspase-3-cleaved tau, is thought to precede tau phosphorylation (Cotman et al., 2005), and so this issue is certainly worthy of further investigation. The pathogenic cascade proposed by Nicholson and Ferreira (2009), together with other $A \beta$-induced events, is shown in Figure 1.

Third, Nicholson and Ferreira (2009) refer to tau toxicity; however, a direct neurotoxic effect of the calpain-cleaved 17 $\mathrm{kDa}$ tau species has not been shown in this study. Although the neuronal death observed is associated with $A \beta$-induced calpain

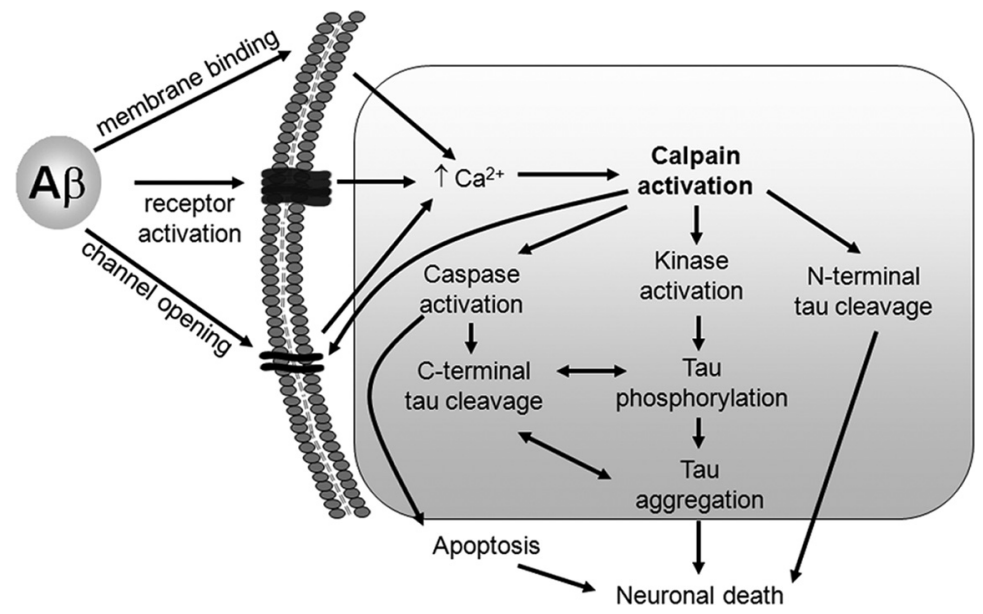

Figure 1. Involvement of multiple pathways in $A \beta$-induced neurodegeneration. Extracellular $A \beta$ induces increased intraneuronal calcium through several different mechanisms, including those represented in this summary. Calpain activation is linked to both $\mathrm{N}$ - and C-terminal tau cleavage and the activation of tau kinases. Caspase-cleaved tau is more likely to become aggregated and highly phosphorylated, all or some of which may result in the induction of neuronal death pathways, including apoptosisassociated caspase activation.

activation, calpain is involved in several different neurodegenerative pathways. Calpain induces aberrant processing of APP, activation of key protein kinases, processing of the synaptic vesicle recycling protein, dynamin-1, inactivation of NMDA receptors involved in learning and memory, such as NR2B, and disruption of plasma membrane ion channels. As an example of the latter, members of the plasma membrane calcium ATPase family remove excess calcium from neurons and thus maintain calcium homeostasis. Several groups have now shown that $\mathrm{A} \beta$ induces calpain-mediated cleavage of ion channels, causing their inactivation and resulting in excess intraneuronal calcium and subsequent excitotoxic cell death. In the cell culture system used by Nicholson and Ferreira (2009), there is likely to be significant cleavage of membrane calcium channels by calpain in the mature neurons, which, regardless of tau cleavage, will certainly contribute to the $\mathrm{A} \beta$ induced neuronal death observed in this study.

A fourth confound that complicates interpretation of Nicholson and Ferreira's (2009) results is the effects of membrane cholesterol depletion on lipid rafts/detergentresistant membranes. Cholesterol and sphingolipids are enriched in lipid rafts, which function as signal transduction platforms in neurons, and are a major site of A $\beta$ production (Hung et al., 2008). Several laboratories have demonstrated that key neuronal proteins involved in calcium transport and signaling are also localized in these compartments. Depleting cholesterol from membranes alters the protein composition of lipid rafts, disturbing membrane trafficking, calcium transporter activity, and related functions. Thus, it is important to know whether the effects on tau described by Nicholson and Ferreira (2009) are mediated specifically by altered lipid raft function after reduction of membrane cholesterol. Interestingly, the human Prion Protein $\left(\operatorname{PrP}^{\mathrm{c}}\right)$ has recently been shown to be important in $\mathrm{A} \beta$ mediated synaptic plasticity as it acts as a receptor for $A \beta$ (Laurén et al., 2009). It would be intriguing to determine whether membrane cholesterol influences recruitment of $\operatorname{PrP}^{c}$ to the cell membrane and whether the generation of $17 \mathrm{kDa}$ tau is dependent on the $\mathrm{A} \beta-\operatorname{PrP}^{\mathrm{c}}$ interaction, a question that could be addressed by replicating the current study in $\operatorname{PrP}^{\mathrm{c}}$ knockout neuronal cultures.

Finally, Nicholson and Ferreira (2009) appear to have selected suboptimal conditions for treating neuronal cultures with $\mathrm{A} \beta$. There is now a consensus in the field that soluble, oligomeric $\mathrm{A} \beta$ is more toxic than the fibrillar form used here (Glabe and Kayed, 2006), and the authors have themselves previously reported that soluble oligomeric, and not fibrillar, $A \beta$ leads to a sustained calcium influx and calpain activation that is linked to synaptic loss and neurodegeneration (Kelly and Ferreira, 2006). Aggregated preparations of $\mathrm{A} \beta$ are likely to be heterogeneous in nature, and thus it would be worthwhile to characterize the precise nature of the aggregates used, for example, by size-exclusion chromatography and/or electron microscopy. This would allow determination of the subpopulation of $\mathrm{A} \beta$ species responsible for producing the 
observed effects and comparison with the results of other laboratories. Another interesting consideration in relation to this study is that soluble and aggregated $\mathrm{A} \beta$ have differing membrane-binding capabilities, with soluble, intermediate-sized species of $\mathrm{A} \beta$ binding strongly to lipid membranes, an event required for $\mathrm{A} \beta$-induced neurotoxicity (Hung et al., 2008). Thus, the authors' observation of increased cholesterol in mature neurons might represent an increased binding surface for $A \beta$, contributing to the neuronal death observed only after maturation of neurons in culture.

In summary, the recent paper by Nicholson and Ferreira (2009) is an interesting study that describes a possible relationship between membrane cholesterol and $\mathrm{A} \beta$ induced tau neurotoxicity. Although high cholesterol is viewed as a risk factor for $\mathrm{AD}$ and cholesterol-lowering agents are the focus of intensive research, the involvement of calpain-mediated tau cleavage in disease pathogenesis remains unclear. The development of transgenic animals expressing other fragments of tau derived from $\mathrm{AD}$ brain and cleaved huntingtin species has provided robust support for the involvement of specific protein fragments in AD and Huntington's disease, respectively, and a similar approach using calpain-cleaved tau fragments could provide substantial proof that these are also involved in disease pathogenesis. The development of an animal model expressing the $17 \mathrm{kDa}$ tau fragment would allow the authors to more accurately examine the effects of aging, and dietary cholesterol could be modified to clarify the relationship between age, cholesterol content, and tau-induced neurodegeneration. Such studies will provide further insights into basic disease processes in AD and may also open the possibility of new avenues for therapeutic intervention.

\section{References}

Cotman CW, Poon WW, Rissman RA, BlurtonJones M (2005) The role of caspase cleavage of tau in Alzheimer disease neuropathology. J Neuropathol Exp Neurol 64:104-112.

Glabe CG, Kayed R (2006) Common structure and toxic function of amyloid oligomers implies a common mechanism of pathogenesis. Neurology 66[2 Suppl 1]:S74-S78.

Hanger DP, Anderton BH, Noble W (2009) Tau phosphorylation: the therapeutic challenge for neurodegenerative disease. Trends $\mathrm{Mol}$ Med 15:112-119.

Hung LW, Ciccotosto GD, Giannakis E, Tew DJ, Perez K, Masters CL, Cappai R, Wade JD,
Barnham KJ (2008) Amyloid-beta peptide (Abeta) neurotoxicity is modulated by the rate of peptide aggregation: Abeta dimers and trimers correlate with neurotoxicity. J Neurosci 28:11950-11958.

Kelly BL, Ferreira A (2006) beta-Amyloid-induced dynamin 1 degradation is mediated by N-methyl-D-aspartate receptors in hippocampal neurons. J Biol Chem 281:28079-28089.

Laurén J, Gimbel DA, Nygaard HB, Gilbert JW, Strittmatter SM (2009) Cellular prion protein mediates impairment of synaptic plasticity by amyloid- $\beta$ oligomers. Nature 457:1128-1132.

Mercken M, Grynspan F, Nixon RA (1995) Differential sensitivity to proteolysis by brain calpain of adult human tau, fetal human tau and PHF-tau. FEBS Lett 368:10-14.

Nicholson AM, Ferreira A (2009) Increased membrane cholesterol might render mature hippocampal neurons more susceptible to beta-amyloid-induced calpain activation and tau toxicity. J Neurosci 29:4640-4651.

Park SY, Ferreira A (2005) The generation of a $17 \mathrm{kDa}$ neurotoxic fragment: an alternative mechanism by which tau mediates betaamyloid-induced neurodegeneration. J Neurosci 25:5365-5375.

Roberson ED, Scearce-Levie K, Palop JJ, Yan F, Cheng IH, Wu T, Gerstein H, Yu GQ, Mucke L (2007) Reducing endogenous tau ameliorates amyloid beta-induced deficits in an Alzheimer's disease mouse model. Science 316: $750-754$. 